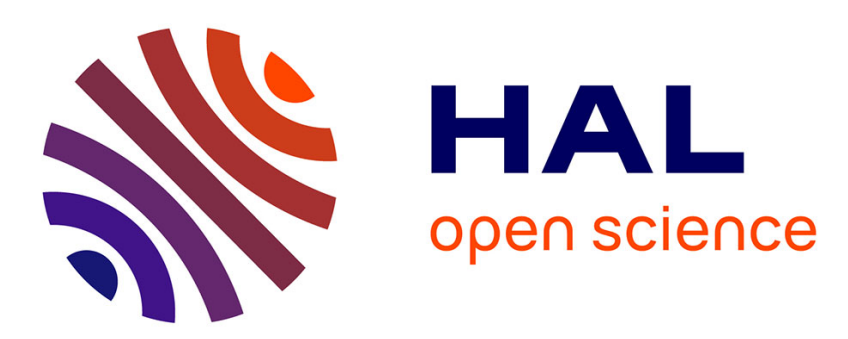

\title{
On the transformation of nonlinear dynamical systems into the extended nonlinear observable canonical form
}

Driss Boutat, Krishna Busawon

\section{To cite this version:}

Driss Boutat, Krishna Busawon. On the transformation of nonlinear dynamical systems into the extended nonlinear observable canonical form. International Journal of Control, 2011, 84 (1), pp.94106. hal-00642797

\section{HAL Id: hal-00642797 https://hal.science/hal-00642797}

Submitted on 18 Nov 2011

HAL is a multi-disciplinary open access archive for the deposit and dissemination of scientific research documents, whether they are published or not. The documents may come from teaching and research institutions in France or abroad, or from public or private research centers.
L'archive ouverte pluridisciplinaire HAL, est destinée au dépôt et à la diffusion de documents scientifiques de niveau recherche, publiés ou non, émanant des établissements d'enseignement et de recherche français ou étrangers, des laboratoires publics ou privés. 


\title{
On the transformation of nonlinear dynamical systems into the extended nonlinear observable canonical form
}

\author{
Driss Boutat $^{\text {a* }}$ and Krishna Busawon ${ }^{\mathrm{b}}$
}

\author{
${ }^{a}$ Institut PRISME, Loire Valley University, ENSI of Bourges, 88 Boulevard de Lahitolle, \\ Bourges 18020, France; ${ }^{b}$ School of Computing, Engineering and Information Sciences, \\ Northumbria University, Newcastle upon Tyne NE1 8ST, UK
}

(Received 27 January 2009; final version received 15 November 2010)

\begin{abstract}
This article deals with the transformation of a class of nonlinear systems into an extended nonlinear observable canonical form (ENOCF) by dynamic extension. The dynamic extension is obtained by adding auxiliary dynamics and virtual outputs to the original system, thus giving rise to a higher dimensional system in an extended state space. Sufficient geometrical conditions to guarantee the existence of a local diffeomorphism which allow the transformation of the extended system into the ENOCF are given. In particular, an algorithm that permits us to compute such a diffeomorphism is derived.
\end{abstract}

Keywords: nonlinear observers; observable canonical form; dynamic extension

\section{Introduction}

Since the beginning of the 1980 s, a number of research work was carried out on the problem of classification of nonlinear dynamical systems that are transformable into simple normal observable forms for which one can design either a Luenberger observer with linear error dynamics or a high gain observer (Bestle and Zeitz 1983; Fliess and Kupka 1983; Krener and Isidori 1983; Krener and Respondek 1985; Keller 1987; Hammouri and Gautier 1988; Xia and Gao 1989; Phelps 1991; Glumineau, Moog, and Plestan 1996; Respondek, Pogromsky, and Nijmeijer 2004). Among these observable forms the most popular one, which is commonly referred to as the nonlinear observer canonical form (NOCF), was addressed by Krener and Isidori (1983). More precisely, consider a single output dynamical system of the following form:

$$
\begin{aligned}
& \dot{x}=f(x), \\
& y=h(x),
\end{aligned}
$$

in a neighbourhood $x \in \mathcal{X} \subset R^{n}$ of 0 and $y \in R$. We assume without loss of generality that $f(0)=0$ and $h(0)=0$. We also assume that system $(1,2)$ satisfies the observability rank condition, i.e. the 1 -forms

$$
\theta_{i}=\mathrm{d} L_{f}^{i-1} h
$$

for $1 \leq i \leq n$ are linearly independent on $\mathcal{X}$. We will call the 1-forms $\left(\theta_{i}\right)_{1 \leq i \leq n}$ the observability 1-forms.

Denote $\theta$ the following multi-valued 1-form:

$$
\theta=\left(\theta_{k}\right)_{1 \leq k \leq n}=\left(\begin{array}{c}
\theta_{1} \\
\theta_{2} \\
\vdots \\
\theta_{n}
\end{array}\right)=\left(\begin{array}{c}
\mathrm{d} h \\
\mathrm{~d} L_{f} h \\
\vdots \\
\mathrm{d} L_{f}^{n-1} h
\end{array}\right) .
$$

Suppose there exists a diffeomorphism $z=\phi(x)$ that permits us to transform system $(1,2)$ into the following form

$$
\begin{gathered}
\dot{z}=A z+\beta(\bar{y}), \\
\bar{y}=\Psi(y)=C z,
\end{gathered}
$$

where $A$ and $C$ are $n \times n$ and $1 \times n$ matrices in the companion observable form: 
and $\Psi$ is a local diffeomorphism. Then, system $(3,4)$ is said to be in the NOCF.

An observer with linear error dynamics for $(3,4)$ is given by

$$
\dot{\hat{z}}=A \hat{z}+\beta(\bar{y})+K(\bar{y}-C \hat{z}),
$$

where the vector $K$ is chosen appropriately such that the error dynamics is stable. In effect, by setting $e=z-\hat{z}$, one can easily check that the error dynamics $\dot{\mathrm{e}}=(A-K C) e$ is linear.

In what follows, we present a version of a result of Krener and Respondek (1985) for single output systems.

Theorem 1.1: There exists a local diffeomorphism that transforms the single output dynamical system $(1,2)$ into the canonical form $(3,4)$ if and only if there exists a function of the output $l(y)$, such that:

$$
\left[\sigma_{i}, \sigma_{j}\right]=0 \quad \text { for all } 1 \leq i, j \leq n,
$$

where the vector field $\sigma_{1}$ is given by

$$
\begin{gathered}
\theta_{k}\left(\sigma_{1}\right)=0 \quad \text { for } 1 \leq k \leq n-1, \\
\theta_{n}\left(\sigma_{1}\right)=l(y) \neq 0,
\end{gathered}
$$

and the vectors fields $\sigma_{i}$, for $2 \leq i \leq n$, are defined recursively as the Lie bracket of $\sigma_{i-1}$ and $f$ as follows:

$$
\sigma_{i}=\left[\sigma_{i-1}, f\right] \text { for } 2 \leq i \leq n .
$$

The above result provides the necessary and sufficient conditions that guarantee the existence of a diffeomorphism $z=\phi(x)$, which permits us to transform system $(1,2)$ into $(3,4)$. It should be noted that the result of Krener and Respondek (1985), originally stated for multi-output systems, is perfectly valid in the case of single output systems.

\section{Remark 1}

- For $l=1$ the above theorem amounts to the result of Krener and Isidori (1983), where $\Psi=I d$ in the form $(3,4)$, which is usually referred to as linearisation by output injection.

- The more general form $(3,4)$ with a diffeomorphism on the output was introduced by

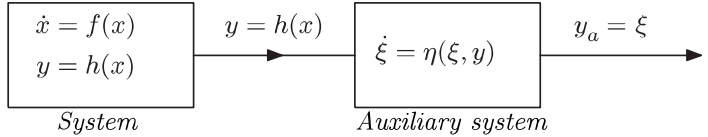

Figure 1. Dynamic extension.

Recently, several authors have considered the so-called dynamic immersion technique to tackle the problem of transforming dynamical systems into a general NOCF (Jouan 2003; Noh, Jo, and Seo 2004; Back, Yu, and Seo 2005, 2006; Boutat 2007). This technique consists in appending, to the dynamical system under study, an auxiliary dynamic $\dot{\xi}=\eta(y, \xi)$ depending on the output and a set of auxiliary states $\xi \in R^{d}$ as illustrated in Figure 1 .

The extended system can be formed as follows:

$$
\left\{\begin{array}{l}
\dot{x}=f(x) \\
\dot{\xi}=\eta(y, \xi) \\
y=h(x) \\
y_{a}=\left(\xi_{1}, \ldots, \xi_{d}\right)^{T},
\end{array}\right.
$$

where

$$
\eta=\left(\eta_{1}, \ldots, \eta_{d}\right)^{T}=\sum_{i=1}^{d} \eta_{i}(y, \xi) \frac{\partial}{\partial \xi_{i}}
$$

is the auxiliary vector field such that its $i$ th component $\eta_{i}=\eta_{i}\left(y, \xi_{1}, \ldots, \xi_{i}\right)$ depends only on the output and the auxiliary variables $\xi_{j}$ for $1 \leq j \leq i$. The main idea is therefore to find a diffeomorphism $z=\phi(x, \xi)$ to linearise the dynamical system (10) in the extended space modulo a vector field, depending on the output and auxiliary states, in the following manner:

$$
\left\{\begin{array}{l}
\dot{z}=\bar{A} z+\beta(\bar{y}, \xi) \\
\bar{y}=\Psi(y, \xi)=z_{n} \\
y_{a}=\xi=\left(\xi_{1}, \ldots, \xi_{d}\right)^{T}
\end{array}\right.
$$

where $z=\left(z_{1}, \ldots, z_{n+d}\right)^{T} \in R^{n+d}$, and $\bar{A}$ is a $(n+d) \times$ $(n+d)$ augmented matrix defined as

$$
\bar{A}=\left(\begin{array}{cc}
A & \mathbf{0}_{n \times d} \\
\mathbf{0}_{d \times n} & \mathbf{0}_{d \times d}
\end{array}\right)
$$

with $A$ being the constant $(n \times n)$ companion matrix defined in (5) and $\beta(y, \xi)=\left(\beta_{1}(y, \xi), \ldots, \beta_{n+d}(y, \xi)\right)^{T}$ is a vector field which depends on the outputs. We call this canonical form the $d$-Extended Nonlinear Observer Canonical Form ( $d$-ENOCF). It is obvious that an observer with linear error dynamics can be also designed for such a canonical form. Since $(3,4)$ is the special case of the $d$-ENOCF (11) when $d=0$, we call $(3,4)$ the 0 -ENOCF. this reason that the theorem of KrenerRespondek is valid in such a case. 
Note that, in practice, the auxiliary system can be viewed as a nonlinear filter which is added to the output of the original system. The output $y_{a}$ can be seen as the filtered version of the output $y$. In the special case where $d=1$ and $\eta(y, \xi)=y$, the observer amounts to the design of a proportional integral (PI) observer (Busawon and Kabore 2001).

An observer for the estimation of the variables $\left(z_{1}, \ldots, z_{n}\right)$ of the dynamical system (11) is given by:

$$
\left(\begin{array}{c}
\dot{\hat{z}}_{1} \\
\dot{\hat{z}}_{2} \\
\vdots \\
\hat{\hat{z}}_{n}
\end{array}\right)=A\left(\begin{array}{c}
\hat{z}_{1} \\
\hat{z}_{2} \\
\vdots \\
\hat{z}_{n}
\end{array}\right)+\left(\begin{array}{c}
\beta_{1}\left(\bar{y}, y_{a}\right) \\
\beta_{2}\left(\bar{y}, y_{a}\right) \\
\vdots \\
\beta_{n}\left(\bar{y}, y_{a}\right)
\end{array}\right)+K(\bar{y}-\hat{\bar{y}}),
$$

where $\hat{\bar{y}}$ is the estimated output. The auxiliary variables $\xi$ are considered as known inputs and the vector $K$ is chosen appropriately such that the error dynamics is stable. Assume that the auxiliary dynamics $\dot{\xi}=\eta(y, \xi)$ is stable in order to ensure the boundedness of $\xi$ which will be emphasised in Example 3.3.

Under some assumptions, Back et al. (2005) proposed some conditions, in terms of a set of partial differential equations, that permit to trans-

135 form the extended system (10) into another canonical form, for which a different observer is proposed. However, the characterisation of this problem in terms of geometrical conditions is still lacking. This leads to the following question ${ }^{1}$ : Are there some geometrical conditions that permit to decide under which circumstances system (10) can be transformed into (11)?

In this article, we address the above question and provide a set of sufficient geometrical conditions that allows to decide whether there exists a diffeomorphism permitting to transform (10) into (11) when $\eta(y, \xi)$ possesses a special structure. First of all, we propose a dual version of Theorem 1.1. This will automatically lead to the development of a procedure to determine

150 whether a 1-ENOCF is realisable. Finally, we provide an algorithm to calculate the diffeomorphism that permits us to transform system $(1,2)$ into the $d$-ENOCF.

This article is organised as follows. In Section 2, we propose a dual version of the commutativity condition (7) of Theorem 1.1. Section 3 is devoted to studying the 1-ENOCF. In Section 4, we give an algorithm that permits us to determine whether or not dynamical system $(1,2)$ can be transformed in the proposed form the state space is of dimension $n$ then the dimension of extended state-space cannot exceed $n-2$. In order not to overload this article, some technical results and proofs are given in the appendix.

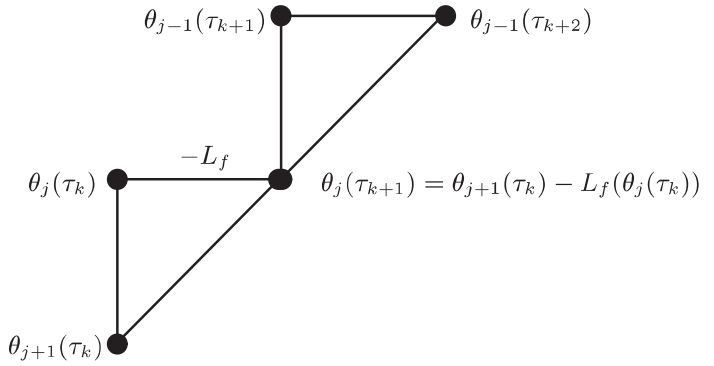

Figure 2. Schematic explanation for triangular structure of $\Lambda$.

\section{A dual version of Theorem 1.1}

In this section, we propose a dual version of Theorem 1.1. In the first version of Theorem 1.1, Krener-Isidori sought a vector field $\tau_{1}$ which satisfies Equations $(8,9)$ with $l(y)=1$. Then, by recursion they obtained the vector fields $\tau_{i}=\left[\tau_{i-1}, f\right]$ for $2 \leq i \leq n$. For simplicity, we shall call the family of vector fields $\left(\tau_{1}, \ldots, \tau_{n}\right)=\tau$ the Krener and Isidori frame. The importance of considering such a frame stems from the fact the Lie brackets $\left[\tau_{i}, \tau_{n}\right]$ for $1 \leq i \leq n-1$ contain all the necessary information to transform a system into the the NOCF or ENOCF.

In fact, the vector field $\sigma_{1}$ of Theorem $1.1 \mathrm{can}$ be computed from the first vector field $\tau_{1}$ of Krener and Isidori frame by setting $\sigma_{1}=l(y) \tau_{1}$ where the function $l(y) \neq 0$ is to be determined, by recursion we have

$$
\sigma_{i}=\left[\sigma_{i-1}, f\right] \text { for } 2 \leq i \leq n .
$$

Consequently, we can derive a relationship between these two frames as follows.

Claim 2.1: The following formulae give the relationship between the frame $\sigma$ and $\tau$ :

$$
\begin{gathered}
\sigma_{2}=l \tau_{2}-\left(L_{f} l\right) \tau_{1}, \\
\sigma_{k}=\sum_{i=0}^{k-1}(-1)^{i} \Gamma_{k}^{i}\left(L_{f}^{i} l\right) \tau_{k-i} \quad \text { for } 2 \leq k \leq n,
\end{gathered}
$$

where

$$
\begin{aligned}
& \Gamma_{k}^{0}=\Gamma_{k}^{k-1}=1 \\
& \Gamma_{k}^{i}=\Gamma_{k-1}^{i-1}+\Gamma_{k-1}^{i} \quad \text { for } 1 \leq i \leq k-2 \\
& \Gamma_{k}^{i}=0 \quad \text { for } i \geq k
\end{aligned}
$$

Proof: The proof of this claim is given in the appendix.

From $(8,9)$ we have $\theta_{n}\left(\sigma_{1}\right)=l(y)$. As $\theta_{n-1}\left(\sigma_{1}\right)=0$, then following diagram of Figure 2 of the appendix, we have $\theta_{n-1}\left(\sigma_{2}\right)=l(y)$. Therefore, one can deduce the following $n \times n$ matrix:

$$
\Lambda=\theta \sigma=\left(\theta_{i}\left(\sigma_{j}\right)\right)_{1 \leq i, j \leq n}=\left(\Lambda_{i j}\right)_{1 \leq i, j \leq n},
$$


which has the following triangular-like structure:

$$
\Lambda=\left(\begin{array}{ccccc}
0 & \ldots & \ldots & 0 & l \\
\vdots & & \ddots & l & \Lambda_{2, n} \\
\vdots & \ddots & \ddots & \ddots & \vdots \\
0 & l & & \ddots & \vdots \\
l & \Lambda_{n, 2} & \ldots & \ldots & \Lambda_{n, n}
\end{array}\right)
$$

and thus is invertible. As a result, we can define the 195 following multi-valued 1 -form:

$$
\omega=\Lambda^{-1} \theta=\left(\omega_{i}\right)_{1 \leq i \leq n} .
$$

The 1-forms $\left(\omega_{i}\right)_{1 \leq i \leq n}$ can be computed recursively as follows:

$$
\omega_{n}=\frac{1}{l} \theta_{1},
$$

$\omega_{n-r}=\frac{1}{l}\left(\theta_{r+1}-\sum_{i=n-r+1}^{n} \Lambda_{r+1, i} \omega_{i}\right)$ for $1 \leq r \leq n-1$.

The following proposition gives the dual version of Theorem 1.1.

Proposition 2.2: The Lie bracket commutativity condition (7) given in Theorem 1.1 is equivalent to the following equality:

$$
\mathrm{d} \omega_{i}=0 .
$$

for all $1 \leq i \leq n$, i.e. the 1 -form $\omega_{i}$ is closed.

Moreover, the ith component of the change of coordinates $z=\phi(x)$ which transforms $(1,2)$ into the 0 -ENOCF $(3,4)$ is given locally by

$$
z_{i}=\phi_{i}(x)=\int_{\gamma} \omega_{i} \quad \text { for } 1 \leq i \leq n,
$$

where

- $\gamma:[0,1] \rightarrow \chi$ is any path such that $\gamma(0)=0$ and $\gamma(1)=x$

- and $\phi_{i}$ is the ith component of the diffeomorphism $\phi(x)$.

Proof: In Technical Formula 1 (Appendix), we set $\nu=\omega, X=\sigma_{i}$ and $Y=\sigma_{j}$. As $\omega\left(\sigma_{k}\right)=\frac{\partial}{\partial z_{k}}$ is constant for all $1 \leq k \leq n$, we have

$$
\mathrm{d} \omega\left(\sigma_{i}, \sigma_{j}\right)=-\omega\left[\sigma_{i}, \sigma_{j}\right] \text { for all } 1 \leq i, j \leq n .
$$

Now, it is easy to show that (7) $\Leftrightarrow(17)$. Indeed, $\sigma$ is a frame of the tangent bundle and $\omega$ is an isomorphism. Therefore, we have $\mathrm{d} \omega=0 \Leftrightarrow\left[\sigma_{i}, \sigma_{j}\right]=0$ for all $1 \leq i, j \leq n$.
As $\mathrm{d} \omega=0$, by Poincaré's theorem (see e.g. Abraham and Marsden 1966), there exists a local diffeomorphism $\phi$ such that $\phi_{*}:=\mathrm{d} \phi=\omega$. In order to see how $\phi$ transforms $f$, we compute $\frac{\partial}{\partial z_{i}} \phi_{*}(f)$.

By using again the Technical Formula 1 with $v=\omega$, $X=\sigma_{i}$ and $Y=f$, we get

$$
\frac{\partial}{\partial z_{i}} \phi_{*}(f)=\phi_{*}\left[\sigma_{i}, f\right]=\phi_{*}\left(\sigma_{i+1}\right)=\frac{\partial}{\partial z_{i+1}},
$$

for all $1 \leq i \leq n-1$ and by integration, we obtain

$$
\phi_{*}(f)=A z+\beta\left(z_{n}\right) .
$$

Now, from (15) and the form of the matrix $\Lambda$, we can see that $\omega_{n}=\mathrm{d} z_{n}=\frac{1}{l} \mathrm{~d} h$. Thus $z_{n}=\Psi(y)$ such that $\Psi^{\prime}(y)=\frac{\mathrm{d}}{\mathrm{d} y} \Psi(y)=\frac{1}{l(y)}$ and $\Psi(0)=0$.

Notice that for $l(y)=1$ we can find the same result as that stated in Nam (1997) which deals with the dual version of Kerner-Isidori theorem by using the same tools. In this case, the coordinates that give the NOCF are $\left(z_{i}\right)_{1 \leq i \leq n}$ with $\mathrm{d} z_{i}=\omega_{i}$ for $1 \leq i \leq n$. In particular, the output $\mathrm{d} y=\omega_{n}=\theta_{1}=\mathrm{d} z_{n}=C$ is linear. Hence, the diffeomorphism linearises the output equally and consequently permits us to design an observer with a linear error dynamics for that class of nonlinear dynamical systems.

One can also refer to Krener and Respondek (1985) or Respondek et al. (2004) to see how such a function $l(y)$ is determined. We will now propose an alternative way to determine the function $l(y)$ whenever it exists. Also, we shall give a technical result that provides the sufficient conditions for the existence of the function $l(y)$. For this, we set $p=$ floor $\left(\frac{n}{2}\right)$ and denote $l^{\prime}(y)=\frac{\mathrm{d} l(y)}{\mathrm{d} y}$.

Lemma 2.3: If there exists a function $l(y)$ such that condition (7) or equivalently condition (17) is satisfied, then

(1) $\left[\tau_{i}, \tau_{k}\right]=0$ for all $1 \leq i \leq p$ and $1 \leq k \leq n-i$,

(2) $\left[\tau_{i}, \tau_{n}\right]-\alpha_{i} \tau_{i} \in \operatorname{span}\left\{\tau_{1}, \ldots, \tau_{i-1}\right\}$ for all $1 \leq i \leq p$, where $\tau_{0}=0$, and $\alpha_{i}(y)$ are some functions of $y$.

(3) for $n=2 p$,

$$
\begin{gathered}
{\left[\tau_{1}, \tau_{k}\right]=0 \quad \text { for } 1 \leq k \leq n-1,} \\
{\left[\tau_{1}, \tau_{n}\right]=\alpha_{1}(y) \tau_{1},} \\
\alpha_{1} l(y)-2 l^{\prime}(y)=0 .
\end{gathered}
$$

(4) for $n=2 p+1$,

$\left[\tau_{1}, \tau_{n}\right]=\left[\tau_{1}, \tau_{k}\right]=\left[\tau_{2}, \tau_{k}\right]=0 \quad$ for $1 \leq k \leq n-1$,

$$
\begin{gathered}
{\left[\tau_{2}, \tau_{n}\right]-\alpha_{2} \tau_{2} \in \operatorname{span}\left\{\tau_{1}\right\},} \\
\alpha_{2} l(y)-n l^{\prime}(y)=0 .
\end{gathered}
$$


Proof: The main objective here is to find a function $l(y)$ such that the frame $\sigma$ is commutative:

$$
\left[\sigma_{i}, \sigma_{j}\right]=0 \text { for all } 1 \leq i, j \leq n .
$$

Consider first the successive Lie derivatives $L_{f}^{i} l$ of $l$ along $f$

$$
\begin{aligned}
& L_{f} l=l^{\prime} L_{f} h, \\
& L_{f}^{2} l=l^{\prime} L_{f}^{2} h+l^{\prime \prime}\left(L_{f} h\right)^{2}, \\
& L_{f}^{3} l=l^{\prime} L_{f}^{3} h+3 l^{(2)} L_{f} h L_{f}^{2} h+l^{(3)}\left(L_{f} h\right)^{3} .
\end{aligned}
$$

and recursively

$$
L_{f}^{n-1} l=l^{\prime} L_{f}^{n-1} h+\sum_{i=0}^{n-2}\left(\gamma_{i}\left(l, l^{\prime}, \ldots, l^{(i)}, L_{f} h, \ldots, L_{f}^{i} h\right) L_{f}^{i} h\right),
$$

where $l^{\prime}=\frac{\mathrm{d} l}{\mathrm{~d} y}, l^{(i)}=\frac{\mathrm{d}^{i} l}{\mathrm{~d} y^{i}}$ and $\gamma_{i}$ is a function of the internal variables. From these expressions, we obtain

$$
\begin{aligned}
\mathrm{d} L_{f}^{i} l\left(\tau_{1}\right) & =0 \quad \text { for } 0 \leq i \leq n-2, \\
\mathrm{~d} L_{f}^{n-1} l\left(\tau_{1}\right) & =l^{\prime} .
\end{aligned}
$$

From $(13,14)$ of Claim 2.1, we have $\sigma_{1}=l \tau_{1}$, $\sigma_{2}=l \tau_{2}-\left(L_{f} l\right) \tau_{1}$ and for $2 \leq k \leq n$

$$
\sigma_{k}=\sum_{i=0}^{k-1}(-1)^{i} \Gamma_{k}^{i}\left(L_{f}^{i} l\right) \tau_{k-i} .
$$

Then $\left[\sigma_{1}, \sigma_{k}\right]=\sum_{i=0}^{k-1}\left[l \tau_{1},(-1)^{i} \Gamma_{k}^{i}\left(L_{f}^{i} l\right) \tau_{k-i}\right]$, and

$$
\begin{aligned}
& {\left[l \tau_{1},(-1)^{i} \Gamma_{k}^{i}\left(L_{f}^{i} l\right) \tau_{k-i}\right]} \\
& \quad=(-1)^{i}\left(\Gamma_{k}^{i} l\left(L_{\tau_{1}} L_{f}^{i} l\right) \tau_{k-i}+\Gamma_{k}^{i} l\left(L_{f}^{i} l\right)\left[\tau_{1}, \tau_{k-i}\right]\right. \\
& \left.\quad-\Gamma_{k}^{i}\left(L_{f}^{i} l\right)\left(L_{\tau_{k-i}} l\right) \tau_{1}\right) .
\end{aligned}
$$

Since $L_{\tau_{k-i}} l=L_{\tau_{k-i-1}} L_{f} l=\cdots=L_{\tau_{1}} L_{f}^{k-i-1} l$, thus for $2 \leq k \leq n$ and $1 \leq i \leq k-1$ we have

$$
\begin{aligned}
L_{\tau_{1}} L_{f}^{i} l & = \begin{cases}l^{\prime}, & \text { if } i=n-1 \\
0, & \text { otherwise }\end{cases} \\
L_{\tau_{k-i}} l & = \begin{cases}l^{\prime}, & \text { if } k-i-n=0 \\
0, & \text { otherwise. }\end{cases}
\end{aligned}
$$

Consequently, for $2 \leq k<n$ we obtain

$$
\left[\sigma_{1}, \sigma_{k}\right]=\sum_{i=0}^{k-1} \Gamma_{k}^{i} l\left(L_{f}^{i} l\right)\left[\tau_{1}, \tau_{k-i}\right],
$$

which shows implicitly that the requirement of $\left[\sigma_{1}, \sigma_{k}\right]=0$ is equivalent to $\left[\tau_{1}, \tau_{k}\right]=0$ for $1 \leq k \leq n-1$.

Then, by using the Jacobi identity, for $1 \leq k \leq n-1$ we get

$$
\left[\tau_{1}, \tau_{k}\right]=\left[\tau_{1},\left[\tau_{k-1}, f\right]\right]=-\left[f,\left[\tau_{1}, \tau_{k-1}\right]\right]+\left[\tau_{k-1}, \tau_{2}\right],
$$

thus $\left[\tau_{2}, \tau_{k-1}\right]=0$. Recursively one has

$$
\left[\tau_{i}, \tau_{k}\right]=0 \quad \text { for } 1 \leq i \leq p \quad \text { and } \quad 1 \leq k \leq n-i,
$$

thus we proved the first condition of this lemma.

When $k=n$, since $L_{\tau_{1}} L_{f}^{k-1} l=L_{\tau_{k-1}} l=l^{\prime}$, then

$$
\begin{aligned}
{\left[\sigma_{1}, \sigma_{n}\right]=} & \sum_{i=0}^{n-1} \Gamma_{n}^{i} l\left(L_{f}^{i} l\right)\left[\tau_{1}, \tau_{n-i}\right] \\
& +\left(-\Gamma_{n}^{0}+(-1)^{n-1} \Gamma_{n}^{n-1}\right) l l^{\prime} \tau_{1} \\
= & \Gamma_{n}^{0} l^{2}\left[\tau_{1}, \tau_{n}\right]+\left(-\Gamma_{n}^{0}+(-1)^{n-1} \Gamma_{n}^{n-1}\right) l l^{\prime} \tau_{1} .
\end{aligned}
$$

As $\Gamma_{n}^{0}=\Gamma_{n}^{n-1}=1$, this leads to

$$
\left[\sigma_{1}, \sigma_{n}\right]=l^{2}\left[\tau_{1}, \tau_{n}\right]+\left(-1+(-1)^{n-1}\right) l l^{\prime} \tau_{1} .
$$

As $\left[\sigma_{1}, \sigma_{n}\right]=0$ and $l$ is a function of $y,(25)$ imposes that $\left[\tau_{1}, \tau_{n}\right]=\alpha_{1}(y) \tau_{1}$ and

$$
\alpha_{1} l(y)-\left(-1+(-1)^{n-1}\right) l^{\prime}=0 .
$$

Let us now examine the Lie bracket $\left[\sigma_{2}, \sigma_{n}\right]$. For this, let us consider

$$
\begin{aligned}
{\left[l \tau_{2}-\right.} & \left.L_{f} l \tau_{1},(-1)^{i} \Gamma_{k}^{i}\left(L_{f}^{i} l\right) \tau_{k-i}\right] \\
= & (-1)^{i}\left(\Gamma_{k}^{i} l\left(L_{\tau_{2}} L_{f}^{i} l\right) \tau_{k-i}+\Gamma_{k}^{i} l\left(L_{f}^{i} l\right)\left[\tau_{2}, \tau_{k-i}\right]\right. \\
& \left.-\Gamma_{k}^{i}\left(L_{f}^{i} l\right)\left(L_{\tau_{k-i}} l\right) \tau_{2}\right)-(-1)^{i}\left(\Gamma_{k}^{i} L_{f} l\left(L_{\tau_{1}} L_{f}^{i} l\right) \tau_{k-i}\right. \\
& \left.+\Gamma_{k}^{i} L_{f} l\left(L_{f}^{i} l\right)\left[\tau_{1}, \tau_{k-i}\right]-\Gamma_{k}^{i}\left(L_{f}^{i} l\right)\left(L_{\tau_{k-i}} L_{f} l\right) \tau_{1}\right) .
\end{aligned}
$$

Since $\left[\tau_{i}, \tau_{k}\right]=0$ for $1 \leq i \leq 2$ and $1 \leq k \leq n-i$, by using the same argument, one obtains

$$
\begin{aligned}
{\left[\sigma_{2}, \sigma_{n}\right]=} & \Gamma_{n}^{0} l^{2}\left[\tau_{2}, \tau_{n}\right]-\Gamma_{n}^{0} l L_{f} l\left[\tau_{1}, \tau_{n}\right] \\
& +\left(-\Gamma_{n}^{0}+(-1)^{n-2} \Gamma_{n}^{n-2}\right) l l^{\prime} \tau_{2} \\
& +\left((-1)^{n-1} \Gamma_{n}^{n-1} l L_{\tau_{1}} L_{f}^{n} l-(-1)^{n-1} \Gamma_{n}^{n-1} L_{f} l l^{\prime}\right. \\
& \left.-\Gamma_{n}^{0} l L_{\tau_{1}} L_{f}^{n} l-\Gamma_{n}^{1} L_{f} l l^{\prime}-\Gamma_{n-1}^{0} l l^{\prime}\right) \tau_{1} .
\end{aligned}
$$

According to the definition of $\Gamma_{k}^{i}$, we have $\Gamma_{n}^{0}=1$ and $\Gamma_{n}^{n-2}=n-1$. If $\left[\sigma_{2}, \sigma_{n}\right]=0$, then the above equation can be written as

$$
l^{2}\left[\tau_{2}, \tau_{n}\right]-\left(1+(-1)^{n-1}(n-1)\right) l \tau_{2}+\rho \tau_{1}=0
$$

with $\rho$ being a function of the states, which implies that

$$
\left[\tau_{2}, \tau_{n}\right]-\alpha_{2}(y) \tau_{2} \in \operatorname{span}\left\{\tau_{1}\right\}
$$

and

$$
\alpha_{2}(y) l(y)-\left(1+(-1)^{n-1}(n-1)\right) l^{\prime}(y)=0 .
$$

Recursively, we can prove that

$$
\left[\tau_{i}, \tau_{n}\right]-\alpha_{i}(y) \tau_{i} \in \operatorname{span}\left\{\tau_{1}, \ldots, \tau_{i-1}\right\} .
$$


Finally, let us see how to determine the function $l(y)$. If $n=2 p$, then $l(y)$ can be determined by (26), i.e. $\quad \alpha_{1}(y) l(y)-2 l^{\prime}(y)=0$. When $n=2 p+1$, since $\left[\tau_{1}, \tau_{n}\right]=0$ implying that $\alpha_{1}=0$, thus it cannot be used to calculate $l(y)$. However, in this case (27) is equal to

$$
\alpha_{2}(y) l(y)-n l^{\prime}(y)=0,
$$

which can be employed to determine $l(y)$.

Remark 2: Since the determination of function $l(y)$ depends on the parity of the dimension $n$, and adding one auxiliary dynamics will make the even (or odd) dimension for original system to odd (or even) one for extended system, thus one might wonder about the essential link between these two cases. However, this is still an open problem, and the following gives only a brief explanation for these two cases.

For this, assume that the dynamical system $(1,2)$ is of dimension equal to $n$, and consider the following augmented system:

$$
\dot{x}=f(x),
$$

$$
\begin{gathered}
\dot{\zeta}=\Psi(y), \\
y_{1}=\zeta,
\end{gathered}
$$

whose dimension is equal to $n+1$. Let $\tilde{\tau}$ be the KrenerIsidori frame of (28)-(30), then the function $\Psi(y)$ is determined in such a way that the first vector filed $\tilde{\tau}_{1}$ of $\tilde{\tau}$ or the second vector field $\tilde{\tau}_{2}$ of $\tilde{\tau}$ commutes with the last vector field $\tilde{\tau}_{n+1}$ of $\tilde{\tau}$ according to the parity of $n$.

For the extended system, we set $\tilde{\theta}_{1}=\mathrm{d} \zeta$, then $\tilde{\theta}_{2}=\Psi^{\prime}(y) \mathrm{d} y=\frac{1}{l} \mathrm{~d} y$ and so on. In such a way, the new frame is given by its first vector field $\tilde{\tau}_{1}=\frac{1}{\Psi^{\prime}(y)} \tau_{1}=$ $l \tau_{1}=\sigma_{1}$, where $\tau_{1}$ is the Krener-Isidori frame for the unextended system $(1,2)$. From $\tilde{\tau}_{1}$, we can easily construct the Krener-Isidori frame for the extended system (28)-(30) as follows:

$$
\begin{aligned}
\tilde{\tau}_{2}= & {\left[\tilde{\tau}_{1}, f+\Psi(y) \frac{\partial}{\partial \zeta}\right]=\left[\tilde{\tau}_{1}, f\right]=\left[\sigma_{1}, f\right]=\sigma_{2}, } \\
\tilde{\tau}_{i}= & {\left[\tilde{\tau}_{i-1}, f+\Psi(y) \frac{\partial}{\partial \zeta}\right]=\left[\tilde{\tau}_{i-1}, f\right]=\left[\sigma_{i-1}, f\right]=\sigma_{i}, } \\
& \text { for } 2 \leq i \leq n
\end{aligned}
$$

and

$$
\tilde{\tau}_{n+1}:=\left[\tilde{\tau}_{n}, f+\Psi(y) \frac{\partial}{\partial \zeta}\right]=\frac{\partial \Psi(y)}{\partial y} \frac{\partial}{\partial \zeta}+\left[\tilde{\tau}_{n}, f\right]
$$

since $\mathrm{d} \Psi(y)\left(\tilde{\tau}_{i}\right)=\frac{\partial \Psi(y)}{\partial y} \mathrm{~d} y\left(\tilde{\tau}_{i}\right)=0$ for $2 \leq i \leq n-1$ and $\mathrm{d} \Psi(y)\left(\tilde{\tau}_{n}\right)=\frac{\partial \Psi(y)}{\partial y} \mathrm{~d} y\left(\tilde{\tau}_{n}\right)=\frac{\partial \Psi(y)}{\partial y}$. Consequently, by using the Jacobi identity, we get

$$
\begin{aligned}
{\left[\tilde{\tau}_{1}, \tilde{\tau}_{n+1}\right] } & =\left[\tilde{\tau}_{1}, \frac{\partial \Psi(y)}{\partial y} \frac{\partial}{\partial \zeta}+\left[\tilde{\tau}_{n}, f\right]\right] \\
& =-\left[f,\left[\tilde{\tau}_{1}, \tilde{\tau}_{n}\right]\right]+\left[\tilde{\tau}_{n}, \tilde{\tau}_{2}\right],
\end{aligned}
$$

since $\mathrm{d} \frac{\partial \Psi(y)}{\partial y} \tilde{\tau}_{i}=0$ for $1 \leq i \leq n-1$.

Hence, if $n$ is odd, we must have $\left[\tau_{1}, \tau_{n}\right]=0$, thus by the construction of $\tilde{\tau}_{i}$ we have $\left[\tilde{\tau}_{1}, \tilde{\tau}_{n}\right]=0$, and finally it gives

$$
\left[\tilde{\tau}_{1}, \tilde{\tau}_{n+1}\right]=\left[\tilde{\tau}_{n}, \tilde{\tau}_{2}\right]=\left[\sigma_{n}, \sigma_{2}\right]
$$

which exhibits the relation between the extended system and unextended system with odd dimension.

When $n$ is even, by the construction of $\tilde{\tau}_{i}$, since we must have $\left[\tilde{\tau}_{1}, \tilde{\tau}_{n+1}\right]=0$, this yields $\left[\tilde{\tau}_{n}, \tilde{\tau}_{2}\right]=$ $\left[\sigma_{n}, \sigma_{2}\right]=0$, thus

$$
\begin{aligned}
{\left[\tilde{\tau}_{2}, \tilde{\tau}_{n+1}\right] } & =\left[\tilde{\tau}_{2},\left[\tilde{\tau}_{n}, f\right]+\frac{\partial \Psi(y)}{\partial y} \frac{\partial}{\partial \zeta}\right] \\
& =\left[\tilde{\tau}_{2},\left[\tilde{\tau}_{n}, f\right]\right]+\left[\tilde{\tau}_{2}, \frac{\partial \Psi(y)}{\partial y} \frac{\partial}{\partial \zeta}\right] \\
& =-\left[f,\left[\tilde{\tau}_{2}, \tilde{\tau}_{n}\right]\right]+\left[\tilde{\tau}_{n},\left[\tilde{\tau}_{2}, f\right]\right]+\left[\tilde{\tau}_{2}, \frac{\partial \Psi(y)}{\partial y} \frac{\partial}{\partial \zeta}\right] \\
& =-\left[f,\left[\sigma_{2}, \sigma_{n}\right]\right]+\left[\sigma_{n}, \sigma_{3}\right]+\left[\tilde{\tau}_{2}, \frac{\partial \Psi(y)}{\partial y} \frac{\partial}{\partial \zeta}\right] \\
& =\left[\sigma_{n}, \sigma_{3}\right],
\end{aligned}
$$

which can be used to determine $l(y)$ for the unextended system $(1,2)$ with even dimension.

\section{Remark 3}

- The Krener-Isidori frame $\tau$ and the Lie brackets of $\left[\tau_{1}, \tau_{n}\right]$ are well determined and known. More precisely,

$$
\left[\tau_{1}, \tau_{n}\right]=\sum_{i=1}^{n} \mu_{i}(x) \tau_{i},
$$

with the functions $\mu_{i}$ being well known. In Lemma 2.3 we, of course, demanded that $\mu_{i}=0$ for $2 \leq i \leq n$ and that $\mu_{1}=\alpha_{1}(y)$ is only a function of the output $y$.

- If $\left[\tau_{1}, \tau_{n}\right]=0$, then from (25), we have $l^{\prime}=0$. In this case, nothing can be done if ever there is one Lie bracket $\left[\tau_{j}, \tau_{n}\right] \neq 0$ for a certain $2 \leq j \leq n-1$.

- From Equations (21) and (24), we can see that

$$
l=e^{\epsilon \int \alpha_{i} \mathrm{~d} y} \quad \text { for } i=1,2,
$$

where $\epsilon$ is either $\frac{1}{2}$ or $\frac{1}{n}$ according to the parity of $n$.

Let us illustrate the above result by considering the following dynamical system. 
Example 2.4: Consider the following dynamical system defined on the state space $\mathcal{X}=R \times] \frac{-\pi}{2}, \frac{\pi}{2}[$ :

$$
\left\{\begin{array}{l}
\dot{x}=\left(\begin{array}{c}
\dot{x}_{1} \\
\dot{x}_{2}
\end{array}\right)=\left(\begin{array}{c}
x_{1} x_{2} \\
x_{1}
\end{array}\right)=f(x) . \\
y=\sin x_{2}=h(x)
\end{array}\right.
$$

The observability 1-forms of the system are:

$$
\begin{aligned}
& \theta_{1}=\mathrm{d} L_{f}^{0} h=\cos x_{2} \mathrm{~d} x_{2} \\
& \theta_{2}=\mathrm{d} L_{f}^{1} h=\cos x_{2} \mathrm{~d} x_{1}-x_{1} \sin x_{2} \mathrm{~d} x_{2}
\end{aligned}
$$

The Krener-Isidori frame $\tau=\left(\tau_{1}, \tau_{2}\right)$ is given by

$$
\begin{aligned}
\tau_{1} & =\frac{1}{\cos x_{2}} \frac{\partial}{\partial x_{1}}, \\
\tau_{2} & =\left[\tau_{1}, f\right]=\frac{1}{\cos x_{2}} \frac{\partial}{\partial x_{2}}+\frac{1}{\cos x_{2}}\left(x_{2}-x_{1} \tan x_{2}\right) \frac{\partial}{\partial x_{1}} .
\end{aligned}
$$

By direct computation, we obtain

$$
\left[\tau_{1}, \tau_{2}\right]=-2\left(\frac{1}{\cos x_{2}}\right)^{2} \tan x_{2} \frac{\partial}{\partial x_{1}} \neq 0 .
$$

Now, we have

$$
\left[\tau_{1}, \tau_{2}\right]=-2 \frac{\sin x_{2}}{\left(\cos x_{2}\right)^{2}} \tau_{1} .
$$

As $n=2$, we use the differential Equation (21) of Lemma 2.3 to deduce that

$$
l=\cos x_{2}=\sqrt{1-y^{2}}
$$

for all $\left.x_{2} \in\right] \frac{-\pi}{2}, \frac{\pi}{2}[$. Therefore,

$$
\begin{aligned}
& \sigma_{1}=l \tau_{1}=\frac{\partial}{\partial x_{1}}, \\
& \sigma_{2}=\frac{\partial}{\partial x_{2}}+x_{2} \frac{\partial}{x_{1}} .
\end{aligned}
$$

Then,

$$
\Lambda=\theta \sigma=\left(\begin{array}{cc}
0, & \cos x_{2} \\
\cos x_{2}, & x_{2} \cos x_{2}-x_{1} \sin x_{2}
\end{array}\right)
$$

and consequently,

$$
\omega=\mathrm{d} z=\left(\begin{array}{c}
\mathrm{d} x_{1}-x_{2} \mathrm{~d} x_{2} \\
\mathrm{~d} x_{2}
\end{array}\right) .
$$

Finally, on the open set $\mathcal{X}$, the dynamical system (31) becomes

$$
\left\{\begin{array}{l}
\dot{z}_{1}=0 \\
\dot{z}_{2}=z_{1}+\frac{1}{2} \bar{y}^{2} \\
\bar{y}=z_{2}
\end{array}\right.
$$

370 for which an observer in the form of (6) can be designed.
Remark 4: Let $\alpha=\alpha_{1}$ or $\alpha=\alpha_{2}$ according to whether $n$ is even or odd.

(i) If condition (1) or (2) in Lemma 2.3 is fulfilled and $\alpha=0$, (i.e. $l$ is constant) but the frame $\sigma$ does not satisfy condition (7), then the 0 -ENOCF problem cannot be solved by Theorem 1.1. We then need to study the 1-ENOCF, which is the main objective of the next section.

(ii) If condition (1) or (2) in Lemma 2.3 is fulfilled and $\alpha \neq 0$, then we have $l(y)$ at our disposition. Moreover, if the frame $\sigma$ does not satisfy condition (7), then we consider the dynamical system issued from $(1,2)$ with the same dynamics (1) and the output $\bar{y}=\int_{0}^{y} \frac{1}{l(s)} \mathrm{d} s$. For this new dynamical system, we have $\alpha=0$ and this brings us back to (i) of this remark with the new $\alpha=0$.

From Lemma 2.3, it is important to emphasise that the fulfilment of Lemma 2.3 is sufficient for finding the function $l(y)$ and the function $\Psi(y)$. We then need to consider the new dynamical system

$$
\begin{aligned}
& \dot{x}=f(x), \\
& \bar{y}=\Psi(y),
\end{aligned}
$$

where we have only changed the output. It is easy to see that the Krener-Isidori frame $\tau$ of system (32) is exactly the frame $\sigma$ of the original system $(1,2)$. If $\sigma$ for $(1,2)$ satisfies the commutativity condition, then system (32) can be put into the NOCF of Krener-Isidori, which is equivalent to saying that system $(1,2)$ can be put into the 0-ENOCF.

In what follows, we shall consider dynamical systems for which the frame $\sigma$ satisfies the conditions of Lemma 2.3, i.e. system of the form described by (32). Consequently, we shall henceforth set $\sigma=\tau$.

\section{Sufficient conditions for 1-ENOCF}

In this section, we consider the extended dynamical system given by (10) when $d=1$ by setting

$$
F=f+\eta_{1}\left(y, \xi_{1}\right) \frac{\partial}{\partial \xi_{1}},
$$

where $\eta_{1}$ depends on $y$ and $\xi_{1}$ and

$$
\dot{\xi}_{1}=\eta_{1}\left(y, \xi_{1}\right) \text {. }
$$

We will specify the function $\eta_{1}$ later on.

One can view each vector field $\tau_{i}$ of the Krener410

Isidori frame as a vector field in the extended space 
by expressing $\tau_{i}$ as

$$
\tau_{i}=\tau_{i}+0 \frac{\partial}{\partial \xi_{1}}
$$

in $R^{n} \times R$.

We now will proceed in the same way as in the previous section. Let us consider again the first vector field $\tau_{1}$ of the Krener-Isidori frame and define the following new frame:

$$
\begin{gathered}
\left.\sigma_{1}^{1}=l\left(\xi_{1}\right) \tau_{1} \text { with } l\left(\xi_{1}\right) \neq 0 \text { on }\right]-r, r[, \\
\sigma_{i}^{1}=\left[\sigma_{i-1}^{1}, F\right] \text { for } 2 \leq i \leq n+1 .
\end{gathered}
$$

N.B. The ' 1 ' in the superscript refers to the dimension $d=1$. We shall later use the notation $\sigma_{i}^{k}$ to define a new frame when $d=k$.

Lemma 3.1: If

$$
\left[\sigma_{i}^{1}, \sigma_{j}^{1}\right]=0 \quad \text { for } 1 \leq i, j \leq n
$$

then

(1) for all $1 \leq i \leq p$ and $1 \leq k \leq n-p+1,\left[\tau_{i}, \tau_{k}\right]=0$;

(2) for all $2 \leq i \leq p,\left[\tau_{i}, \tau_{n}\right]-\alpha_{i} \tau_{i-1} \in \operatorname{span}\left\{\tau_{1}, \ldots\right.$, $\left.\tau_{i-2}\right\}$ with $\tau_{0}=0$ and $\alpha_{i}$ is some function of $y$;

(3) If $n=2 p+1$, then $\alpha_{2}(y) \neq 0$ and

$$
\alpha_{2} l+2 \frac{\partial \eta_{1}}{\partial y} l^{\prime}=0
$$

(4) if $n=2 p$, then $\alpha_{3}(y) \neq 0$ and

$$
\begin{gathered}
{\left[\sigma_{2}, \sigma_{n}\right]=0} \\
\alpha_{3} l+(n+1) l^{\prime} \frac{\partial \eta_{1}}{\partial y}=0 .
\end{gathered}
$$

Moreover, the function $\eta_{1}\left(y, \xi_{1}\right)$ has the following form:

$$
\eta_{1}\left(y, \xi_{1}\right)=\kappa_{1}\left(\xi_{1}\right) \gamma(y)+\kappa_{2}\left(\xi_{1}\right),
$$

where $\kappa_{1}\left(\xi_{1}\right)=\frac{l}{l^{\prime}}, \kappa_{2}\left(\xi_{1}\right)$ is arbitrary and $\gamma$ is the integral of $\frac{-\alpha_{2}}{2}$ or $-\frac{\alpha_{3}}{n+1}$ (according to the parity of $n$ ).

Proof: The proof of this lemma is similar to that for Lemma 2.3, thus we only give the sketch. Let $l\left(\xi_{1}\right) \neq 0$
As for the proof of Claim 2.1, a straightforward induction gives,

$$
\sigma_{2}^{1}=l \tau_{2}-\left(L_{F} l\right) \tau_{1},
$$

$$
\sigma_{k}^{1}=\sum_{i=0}^{k-1}(-1)^{i} \Gamma_{k}^{i} L_{F}^{i} l \tau_{k-i}, \quad \text { for } 3 \leq k \leq n,
$$

where

$$
\begin{aligned}
& \Gamma_{k}^{0}=\Gamma_{k}^{k-1}=1, \\
& \Gamma_{k}^{i}=\Gamma_{k-1}^{i-1}+\Gamma_{k-1}^{i} \quad \text { for } 1 \leq i \leq k-2 .
\end{aligned}
$$

Now, the successive Lie derivatives of function $l$ along the extended vector field $F$ yield

$$
L_{F} l=\eta_{1} l^{\prime}, \quad L_{F}^{2} l=\frac{\partial \eta_{1}}{\partial y} l^{\prime} L_{f} h+\eta_{1} \frac{\partial\left(\eta_{1} l^{\prime}\right)}{\partial \xi_{1}}
$$

and so on, which implies that

$$
L_{\tau_{j}} l\left(\xi_{1}\right)=0 \text { for } 1 \leq j \leq n .
$$

Therefore, from $(40,41)$ we have

$$
\left[\sigma_{1}^{1}, \sigma_{n}^{1}\right]=l^{2}\left[\tau_{1}, \tau_{n}\right]
$$

Then $\left[\sigma_{1}^{1}, \sigma_{n}^{1}\right]=0$ if and only if $\left[\tau_{1}, \tau_{n}\right]=0$.

Now, a straightforward calculation gives

$$
\left[\sigma_{2}^{1}, \sigma_{n}^{1}\right]=l^{2}\left[\tau_{2}, \tau_{n}\right]+\left((-1)^{2}+(-1)^{n-1}\right) l \eta_{1}^{\prime} \tau_{1},
$$

where $\eta_{1}^{\prime}=\frac{\mathrm{d} \eta_{1}}{\mathrm{~d} y}$. If $n=2 p+1$, then $\left[\sigma_{2}^{1}, \sigma_{n}^{1}\right]=0$ implies that $\left[\tau_{2}, \tau_{n}\right]=\alpha_{2}(y) \tau_{1}$ and

$$
\alpha_{2}(y) l+2^{\prime} \frac{\partial \eta_{1}}{\partial y}=0 .
$$

If $n=2 p$, then (42) reduces to $\left[\sigma_{2}^{1}, \sigma_{n}^{1}\right]=l^{2}\left[\tau_{2}, \tau_{n}\right]$. Hence, $\left[\sigma_{2}^{1}, \sigma_{n}^{1}\right]=0$ if and only if $\left[\tau_{2}, \tau_{n}\right]=0$. In this case, we have to consider $\left[\sigma_{3}^{1}, \sigma_{n}^{1}\right]$, which is as follows:

$$
\begin{aligned}
{\left[\sigma_{3}^{1}, \sigma_{n}^{1}\right]=} & l^{2}\left[\tau_{3}, \tau_{n}\right]+\left((-1)^{2}(2)\right. \\
& \left.+(-1)^{n-2}(n-1)\right) l \eta_{1} \tau_{2}+\mu(x) \tau_{1},
\end{aligned}
$$

where $\mu(x)$ is a function of the state. If we impose $\left[\sigma_{3}^{1}, \sigma_{n}^{1}\right]=0$ then we must have $\left[\tau_{3}, \tau_{n}\right]=\alpha_{3}(y) \tau_{2}$ and thus

$$
\alpha_{3}(y) l+(n+1) l^{\prime} \frac{\partial \eta_{1}}{\partial y}=0 .
$$

Finally, from the above equation we can see that,

$$
\frac{\partial \eta_{1}}{\partial y}=\frac{-\alpha_{3}}{n+1} \frac{l}{l^{\prime}} .
$$

Hence,

$$
\eta_{1}\left(y, \xi_{1}\right)=\kappa_{1}\left(\xi_{1}\right) \gamma(y)+\kappa_{2}\left(\xi_{1}\right),
$$

$$
\sigma_{i}^{1}=\left[\sigma_{i-1}^{1}, F\right] \text { for } 2 \leq i \leq n .
$$


where $\kappa_{1}\left(\xi_{1}\right)=\frac{l}{l^{\prime}}, \quad \gamma(y)=\int_{0}^{y} \frac{-\alpha_{2}(s)}{2} \mathrm{~d} s \quad$ if $n=2 p \quad$ and $\gamma(y)=\int_{0}^{y} \frac{-\alpha_{3}(s)}{n+1} \mathrm{~d} s$ if $n=2 p+1$.

We can now state the following theorem:

Theorem 3.2: If there exists a function $l\left(\xi_{1}\right)$ such that condition (35) is satisfied, then there exists a local diffeomorphism $z=\phi\left(x_{e}\right)$ in the extended space which transforms the dynamical system (10) into the following form:

$$
\dot{z}=A z+\beta\left(y, \xi_{1}\right)
$$

$$
\begin{gathered}
\dot{z}_{n+1}=\dot{\xi}_{1}=\kappa_{1}\left(\xi_{1}\right) \gamma(y)+\kappa_{2}\left(\xi_{1}\right), \\
\bar{y}=z_{n}, \\
y_{a}=z_{n+1}=\xi_{1} .
\end{gathered}
$$

Proof: Let $l\left(\xi_{1}\right) \neq 0$ and consider the following vector field $\sigma_{1}^{1}=l \tau_{1}$. From Equations (34) and (41), we get:

$$
\sigma_{n+1}^{1}:=\left[\sigma_{n}^{1}, F\right]=l \frac{\partial \eta_{1}}{\partial y} \frac{\partial}{\partial \xi_{1}}+\sum_{i=1}^{n} \mu_{i}\left(x_{e}\right) \tau_{i}
$$

As $\frac{\partial \eta_{1}}{\partial y} \neq 0$, the frame $\sigma^{1}=\left(\sigma_{i}^{1}\right)_{1 \leq i \leq n+1}$ belongs to the tangent bundle of the extended space. Let us choose a vector field of the form

$$
T_{n+1}=\frac{\partial}{\partial \xi_{1}}+\sum_{i=1}^{n} \nu_{i}\left(x_{e}\right) \tau_{i}
$$

such that

$$
\left[\sigma_{i}^{1}, T_{n+1}\right]=0 \quad \text { for all } 1 \leq i \leq n .
$$

We have

$$
\mathrm{d} h_{a}\left(T_{n+1}\right)=1 .
$$

Now, we consider

$$
\begin{gathered}
\theta_{i}^{1}=\mathrm{d} L_{F}^{i-1} h \quad \text { for } 1 \leq i \leq n, \\
\theta_{n+1}^{1}=\mathrm{d} \xi .
\end{gathered}
$$

Let us also define the following $(n+1) \times(n+1)$ matrix

$$
\Lambda^{1}=\left(\Lambda_{i, j}^{1}\right)_{1 \leq i, j \leq n+1}
$$

where

$$
\begin{aligned}
\Lambda_{i, j}^{1} & =\theta_{i}^{1}\left(\sigma_{j}^{1}\right) \quad \text { for all } 1 \leq j \leq n, \\
\Lambda_{i, n+1}^{1} & =\theta_{i}^{1}\left(T_{n+1}\right) .
\end{aligned}
$$

It easy to see that this matrix has the following form:

$$
\Lambda^{1}=\left(\begin{array}{ccccc}
0 & \cdots & 0 & 0 & 1 \\
\vdots & & 0 & l & \Lambda_{2, n+1}^{1} \\
0 & \ddots & l & * & * \\
0 & l & * & & * \\
l & \Lambda_{n, 2}^{1} & * & \ldots & *
\end{array}\right) .
$$

Proceeding as for the proof of Proposition 2.2, we introduce the following multi 1-forms:

Using similar argument as in the proof of Proposition 2.2, we conclude that

(i) conditions (35) and (49) are equivalent to $\mathrm{d} \omega^{1}=0$.

(ii) condition (35) implies that there exists a diffeomorphism $z=\phi\left(x_{e}\right)$ that transforms the dynamical system (10) into (48-45).

Now, by writing

$$
\frac{\partial}{\partial z_{i}} \phi_{*}(F)=\phi_{*}\left(\left[\sigma_{i}, F\right]\right)=\phi_{*}\left(\sigma_{i+1}\right)=\frac{\partial}{\partial z_{i+1}}
$$

for all $1 \leq i \leq n-1$ we show that $\phi_{*}(F)$ is in the required form.

\section{Remark 5}

(i) As $\eta=\kappa_{1}\left(\xi_{1}\right) \gamma(y)+\kappa_{2}\left(\xi_{1}\right)$ with $\kappa_{2}\left(\xi_{1}\right)$ being arbitrary, the latter can be viewed as known input. Consequently, we use $\kappa_{2}\left(\xi_{1}\right)$ to stabilise $\dot{\mathrm{z}}_{n+1}=\kappa_{1}\left(\xi_{1}\right) \gamma(y)+\kappa_{2}\left(\xi_{1}\right)$ in the sense of Inputto-State Stability (Sontag 1995).

(ii) Note that if the vector fields of the frame $\sigma^{1}=\left(\sigma_{i}^{1}\right)_{1<i<n+1}$ commute, then by setting $T_{n+1}=\sigma_{n+1}^{1}$, we get

where $y_{a}=z_{n+1}=\xi_{1}$.

Let us consider an example to clarify the previous statements.

Example 3.3: We consider the example given in Back et al. (2005) for comparison purposes

$$
\omega^{1}=\left(\Lambda^{1}\right)^{-1} \theta^{1} .
$$

$$
\phi_{*}(F)=A z+\beta\left(\xi_{1}\right),
$$

$$
\left\{\begin{array}{l}
\dot{x}_{1}=-4 x_{3} x_{1}-3 x_{2}^{2}-6 x_{3}^{2} x_{2} \\
\dot{x}_{2}=x_{1} \\
\dot{x}_{3}=x_{2} \\
y=h(x)=x_{3} .
\end{array}\right.
$$


A straightforward computation of the Krener-Isidori frame gives:

$$
\begin{aligned}
\tau_{1} & =\frac{\partial}{\partial x_{1}}, \\
\tau_{2} & =\frac{\partial}{\partial x_{2}}-4 x_{3} \frac{\partial}{\partial x_{1}}, \\
\tau_{3} & =\frac{\partial}{\partial x_{3}}-4 x_{3} \frac{\partial}{\partial x_{2}}+\left(10 x_{3}^{2}-2 x_{2}\right) \frac{\partial}{\partial x_{1}} .
\end{aligned}
$$

Consequently, we have

$$
\begin{aligned}
& {\left[\tau_{1}, \tau_{2}\right]=\left[\tau_{1}, \tau_{3}\right]=0,} \\
& {\left[\tau_{2}, \tau_{3}\right]=2 \tau_{1} .}
\end{aligned}
$$

For this example we have $n=2 p+1$ where $p=1$, then

Condition (4) of Lemma 2.3 is not satisfied. Therefore, we cannot use Theorem 1.1. On the other hand, Conditions (1-3) of Lemma 3.1 are verified, thus yielding

$$
2 \frac{\partial \eta_{1}}{\partial x_{3}} l^{\prime}+2 l=0 .
$$

As it has been mentioned previously, we have

$$
\eta_{1}\left(y, \xi_{1}\right)=\kappa_{1}\left(\xi_{1}\right) \gamma(y)+\kappa_{2}\left(\xi_{1}\right),
$$

with $\gamma(y)=-\int_{0}^{y} \frac{\alpha_{2}}{2}$. If, for simplicity, we choose $\kappa_{1}\left(\xi_{1}\right)=-1$ and $\kappa_{2}\left(\xi_{1}\right)=0$, and it yields $l^{\prime}=-l$. That is $l=e^{-\xi_{1}}$. Therefore, we obtain

$$
\begin{aligned}
& \sigma_{1}^{1}=l \frac{\partial}{\partial x_{1}}, \\
& \sigma_{2}^{1}=l \frac{\partial}{\partial x_{2}}-3 l x_{3} \frac{\partial}{\partial x_{1}}, \\
& \sigma_{3}^{1}=l \frac{\partial}{\partial x_{3}}-2 l x_{3} \frac{\partial}{\partial x_{2}}+\left(3 l x_{3}^{2}-3 x_{2} l\right) \frac{\partial}{\partial x_{1}} .
\end{aligned}
$$

By using the same notations as in the proof of Theorem 3.2, we get

$$
T_{4}=\frac{\partial}{\partial \xi_{1}}-\left(x_{1}-3 x_{2} x_{3}+x_{3}^{3}\right) \frac{\partial}{\partial x_{1}}-\left(x_{2}-x_{3}^{2}\right) \frac{\partial}{\partial x_{2}}-x_{3} \frac{\partial}{\partial x_{3}} .
$$

Finally, we obtain

$$
\Lambda^{1}=\left(\begin{array}{cccc}
0, & 0, & 0, & 1 \\
0, & 0, & l, & -x_{3} \\
0, & l, & -2 l x_{3}, & -\left(x_{2}-x_{3}^{2}\right) \\
l, & -3 l x_{3}, & \left(3 l x_{3}^{2}-3 x_{2} l\right), & -\left(x_{1}-3 x_{2} x_{3}+x_{3}^{3}\right)
\end{array}\right)
$$

and $\omega_{1}=\left(\Lambda^{1}\right)^{-1} \theta^{1}$, which provides us the following diffeomorphism:

$$
z=\phi(x)=\left(\begin{array}{c}
\left(x_{1}+3 x_{2} x_{3}+x_{3}^{3}\right) e^{\xi_{1}} \\
\left(x_{2}+x_{3}^{2}\right) e^{\xi_{1}} \\
x_{3} e^{\xi_{1}} \\
\xi_{1}
\end{array}\right) .
$$

This diffeomorphism is the same diffeomorphism given by Back et al. $(2005,2006)$ obtained by solving a set of partial differential equations.

With the deduced diffeomorphism, (52) can be transformed into

$$
\left\{\begin{array}{l}
\dot{z}_{1}=z_{3}^{4} e^{-3 \xi_{1}} \\
\dot{z}_{2}=z_{1}, \dot{z}_{3}=z_{2} \\
\dot{\xi}_{1}=y=z_{3} e^{-\xi_{1}} \\
\bar{y}=z_{3} \\
y_{a}=\xi_{1},
\end{array}\right.
$$

for which an observer of the form (12) can then be designed.

Obviously, one could choose another $\kappa_{1}\left(\xi_{1}\right)$ and $\kappa_{2}\left(\xi_{1}\right)$ which will produce another diffeomorphism transforming (52) into another form with the dynamics $\dot{\xi}_{1}=\kappa_{1}\left(\xi_{1}\right) \gamma(y)+\kappa_{2}\left(\xi_{1}\right)$. Since $\kappa_{2}\left(\xi_{1}\right)$ is arbitrary, thus if $\gamma(y)$ is bounded, then we can always find a $\kappa_{2}\left(\xi_{1}\right)$ such that this dynamics is bounded, i.e. to choose $\kappa_{2}\left(\xi_{1}\right)$ to make the auxiliary dynamics Input-to-State Stable (see Sontag 1995; Sontag and Wang 1995 for details). $A$ trivial case for this example is to set $\kappa_{1}\left(\xi_{1}\right)=B$ and $\kappa_{2}\left(\xi_{1}\right)=A \xi_{1}$, which brings the dynamics to the linear one: $\dot{\xi}_{1}=A \xi_{1}+B y$ and it is obviously ISS if $y$ is bounded and $A$ is chosen to be stable (Sontag 1995).

Remark 6: If we try to solve the 1-ENOCF problem by considering a function $l\left(y, \xi_{1}\right)$, in Lemma 3.1 , which depends both on the output and on the extended variable, then it easy to see that Lemma 2.3 gives us in the first instance a function $l_{0}$ which depends only on the output $y$. We have therefore two cases to study:

- Either the commutativity condition (35) is fulfilled by frame $\sigma$ (in this case, we obtain a 0 -ENOCF form thanks to Theorem 1.1);

- Or condition (35) is not fulfilled. In this case, we set $\bar{y}=\int \frac{1}{l_{0}} \mathrm{~d} y$. Lemma 3.1 provides a function $l_{1}\left(\xi_{1}\right)$ which depends only on the auxiliary variable $\xi_{1}$ such that $l=l_{0} l_{1}$.

Therefore, the 1-ENOCF problem is a decoupled problem. We must deal with it in two stages: first, we use Lemma 2.3 to determine the new output $\bar{y}$, and then we use Lemma 3.1. If condition (7) is fulfilled, then we say that a 0 to $1 \mathrm{ENOCF}$ is realised or simply a $(0 \rightarrow 1)$-ENOCF is realised.

The following example clarifies the above remark.

Example 3.4: Let us consider again the same dynamical system as in Example 3.3 but with the following output

$$
y=h(x)=e^{x_{3}}-1 .
$$


Then, we have

$$
\begin{aligned}
\tau_{1}= & e^{-x_{3}} \frac{\partial}{\partial x_{1}}, \\
\tau_{2}= & e^{-x_{3}} \frac{\partial}{\partial x_{2}}-e^{-x_{3}}\left(4 x_{3}-x_{2}\right) \frac{\partial}{\partial x_{1}}, \\
\tau_{3}= & e^{-x_{3}} \frac{\partial}{\partial x_{3}}+e^{-x_{3}}\left(2 x_{2}-4 x_{3}\right) \frac{\partial}{\partial x_{2}}+e^{-x_{3}}\left(4\left(4 x_{3}-x_{2}\right) x_{3}\right. \\
& \left.-6 x_{2}-6 x_{3}^{2}+x_{2}\left(4 x_{3}-x_{2}+4\right)-x_{1}\right) \frac{\partial}{\partial x_{1}} .
\end{aligned}
$$

Consequently,

$$
\left[\tau_{1}, \tau_{3}\right]=0 \text { and }\left[\tau_{1}, \tau_{2}\right]=0
$$

As

$$
\left[\tau_{2}, \tau_{3}\right]=e^{-x_{3}}\left(12 x_{3}-3 x_{2}+2\right) \tau_{1}+3 e^{-x_{3}} \tau_{2} \neq \rho \tau_{2},
$$

thus condition (2) of Lemma 3.1 is not fulfilled. However, Conditions (1-4) of Lemma 2.3 are fulfilled.

$$
\left[\tau_{2}, \tau_{3}\right]-3 e^{-x_{3}} \tau_{2} \in \operatorname{span}\left\{\tau_{1}\right\}
$$

which gives $l=e^{x_{3}}$. Now, we consider the following new frame:

$$
\begin{aligned}
& \sigma_{1}=l \tau_{1}=\frac{\partial}{\partial x_{1}}, \\
& \sigma_{2}=\frac{\partial}{\partial x_{2}}-4 x_{3} \frac{\partial}{\partial x_{1}}, \\
& \sigma_{3}=\frac{\partial}{\partial x_{3}}-4 x_{3} \frac{\partial}{\partial x_{2}}+\left(10 x_{3}^{2}-2 x_{2}\right) \frac{\partial}{\partial x_{1}} .
\end{aligned}
$$

A direct calculation gives

$$
\begin{aligned}
& {\left[\sigma_{1}, \sigma_{2}\right]=\left[\sigma_{1}, \sigma_{3}\right]=0,} \\
& {\left[\sigma_{2}, \sigma_{3}\right]=2 \sigma_{1} .}
\end{aligned}
$$

Hence, this new frame verifies conditions (1-3) of Lemma 3.1. By solving

$$
2 \frac{\partial \eta_{1}}{\partial x_{3}} l^{\prime}+2 l=0
$$

with $\frac{\partial \eta_{1}}{\partial x_{3}}=1$, we obtain $l=e^{-\xi_{1}}$. Therefore, we are in the similar case of Example 3.4. That is the dynamical system (11) is linearised by dynamic immersion via a $(0 \rightarrow 1)$-ENOCF.

One can deduce the following result.

580 Corollary 3.5: If the state space is of dimension n, then $d$-ENOCF problem admits a possible solution in the proposed form (11) in an extended space of dimension $n+d$, where $d \leq n-2$. For a dynamical system on a 2-dimensional state space the result is also obtained in

Indeed, the objective is to nullify $\left[\sigma_{d}^{d}, \sigma_{n}^{d}\right]=0$ at each stage. The fact of nullifying $\left[\sigma_{1}, \sigma_{n}\right]=0$ gives a

diffeomorphism on the output. As $\left[\sigma_{n}, \sigma_{n}\right]=0$ there remains $d=n-2$ brackets to annul. Hence, we deduce that if the state space is of dimension $n$, then ENOCF problem admits a possible solution in an extended space of dimension $n+d$ where $d \leq n-2$. This fact implies a restriction that the existence of a solution for $d$-ENOCF with $d \geq n-1$ cannot be determined by the proposed method, while this restriction does not exist in Back et al. (2005, 2006).

Before giving an algorithm to solve the $d$-ENOCF problem, some clarifications of the notations that we shall use henceforth are necessary.

\section{Notations}

- For a function $l_{0}(y), l_{0}^{\prime}$ is the derivative of $l_{0}(y)$ with respect to $y$, i.e. $l_{0}^{\prime}=\frac{\mathrm{d} l_{0}}{\mathrm{~d} y}$.

- For $n \geq 3$ and $1 \leq d \leq n-2, l_{d}\left(\xi_{d}\right)$ represents a function on the extended variable $\xi_{d}$ and $l_{d}^{\prime}=\frac{\mathrm{d} l_{d}}{\mathrm{~d} \xi_{d}}$.

- For $\eta_{k}\left(\xi_{d-1}, \xi_{k}\right), \eta_{k}^{\prime}$ represents the derivative with respect to the first variable, that is $\eta_{k}^{\prime}=$ $\frac{\partial \eta_{k}}{\partial \xi_{d-1}}$. In particular, for $\eta_{1}\left(y, \xi_{1}\right)$ we have $\eta_{1}^{\prime}=\frac{\partial \eta_{1}}{\partial y}$.

Hereafter, we set $F_{0}=f$ and $F_{d}=F_{d-1}+\eta_{d} \frac{\partial}{\partial \xi_{d}}$ for $d \geq 1$.

- We denote $\mathcal{P}_{d}$ the product $\mathcal{P}_{d}=l_{0} l_{1} \ldots$ $l_{d-1} \eta_{0}^{\prime} \cdots \eta_{d-1}^{\prime}$.

Finally, $p=$ floor $\left(\frac{n}{2}\right)$

Assume that we have already constructed the frame $\sigma^{d-1}=\left(\sigma_{i}^{d-1}\right)_{1 \leq i \leq n}$ and set $F_{d}=F_{d-1}+\eta_{d}\left(\xi_{d-1}, \xi_{d}\right) \frac{\partial}{\partial \xi_{n+d}}$ and $l_{d}=l_{d}\left(\xi_{1}\right)$, thus we have

$$
\begin{aligned}
& \sigma_{1}^{d}=l_{d} \sigma_{1}^{d-1}, \\
& \sigma_{2}^{d}=l_{d} \sigma_{2}^{d-1}-\left(L_{F_{d}} l_{d}\right) \sigma_{1}^{d-1}, \\
& \sigma_{k}^{d}=\sum_{i=0}^{k-1}(-1)^{i} \Gamma_{k}^{i}\left(L_{F_{d}}^{i} l_{d}\right) \sigma_{k-i}^{d-1},
\end{aligned}
$$

where $\Gamma_{k}^{0}=\Gamma_{k}^{k-1}=1$ and $\Gamma_{k}^{i}=\Gamma_{k-1}^{i-1}+\Gamma_{k-1}^{i}$ for $1 \leq i \leq k-2$. And straightforward calculations yield

$$
\begin{aligned}
{\left[\sigma_{d+1}^{d}, \sigma_{n}^{d}\right]=} & l_{d}^{2}\left[\sigma_{d+1}^{d-1}, \sigma_{n}^{d-1}\right]+\left((-1)^{d+1}+(-1)^{n-1}\right) \\
& \times \mathcal{P}_{d} \eta_{d}^{\prime} l_{d}^{\prime} \sigma_{d}^{d} \bmod \operatorname{span}\left\{\sigma_{1}^{d}, \ldots, \sigma_{d-1}^{d}\right\}
\end{aligned}
$$

and if $d<n-2$, then we have

$$
\begin{aligned}
{\left[\sigma_{d+2}^{d}, \sigma_{n}^{d}\right]=} & l_{d}^{2}\left[\sigma_{d+2}^{d-1}, \sigma_{n}^{d-1}\right]+\left((-1)^{d+1}(d+1)+(-1)^{n-2}\right. \\
& \times(n-1)) \mathcal{P}_{d} \eta_{d}^{\prime} l_{d}^{\prime} \sigma_{d+1}^{d} \bmod \operatorname{span}\left\{\sigma_{1}^{d}, \ldots, \sigma_{d}^{d}\right\}
\end{aligned}
$$

Using the above equations, we can give an algorithm that will enable us to verify whether or not a dynamic extension of dimension $d$ is possible. 


\subsection{Algorithm for determining the d-ENOCF}

625 Step 1: Construct the Krener-Isidori frame $\tau$; Step 2: If $\left[\tau_{i}, \tau_{j}\right]=0$ for all $1 \leq i, j \leq n$, then go to Step 7. Otherwise construct the Krener-Respondek frame $\sigma$. If $\left[\sigma_{i}, \sigma_{j}\right]=0$ for all $1 \leq i, j \leq n$, then go to Step 7. Otherwise set $d=0$ and $p=$ floor $\left(\frac{n}{2}\right)$.

630 Step 3: If $\left[\sigma_{s+1}^{d}, \sigma_{j}^{d}\right]=\cdots=\left[\sigma_{s+2}^{d}, \sigma_{j}^{d}\right]=0$ for all $1 \leq$ $j \leq n-1$ and $0 \leq s \leq d$ and $n=2 p+1$, then $\left[\sigma_{d+1}^{d}, \sigma_{n}^{d}\right]-$ $\alpha \sigma_{d}^{d} \in \operatorname{span}\left\{\sigma_{1}^{d}, \ldots,{ }_{d-1}^{d}\right\}$, and compute $\left(l_{d}, \eta_{d}\right.$ from

$$
\alpha l_{d}+(-1)^{d+1} 2 \mathcal{P}_{d} \eta_{d}^{\prime} l_{d}^{\prime}=0
$$

and go to Step 5;

Step 4: If

$$
\left[\sigma_{s+1}^{d}, \sigma_{j}^{d}\right]=\cdots=\left[\sigma_{s+2}^{d}, \sigma_{j}^{d}\right]=0
$$

635 for all $1 \leq j \leq n-1$ and $0 \leq s \leq d$ and $n=2 p$, then $\left[\sigma_{d+1}^{d}, \sigma_{n}^{d}\right]=0$ and $\left[\sigma_{d+2}^{d}, \sigma_{n}^{d}\right]-\alpha \sigma_{d+1}^{d} \in \operatorname{span}\left\{\sigma_{1}^{d}, \ldots\right.$, $\left.\sigma_{d}^{\mathrm{d}}\right\}$, and compute $\left(l_{d}, \eta_{d}\right)$ from

$$
\alpha l_{d}+(-1)^{d+1}(n+d) \mathcal{P}_{d} l_{d}^{\prime} \eta_{d}^{\prime}=0
$$

and go to Step 5;

Step 5: Construct the frame $\sigma^{d+1}=\left(\sigma_{i}^{d+1}\right)_{1 \leq i \leq n}$ by

640 setting $\sigma_{1}^{d+1}=l_{d+1} \sigma_{1}^{k} \quad$ and $\quad \sigma_{i}^{d+1}=\left[\sigma_{i-1}^{d+1}, F_{d}\right]$ for $2 \leq 1 \leq n$

Step 6: If $\left[\sigma_{i}^{d+1}, \sigma_{j}^{d+1}\right]=0$ for all $1 \leq i, j \leq n$, then go to Step 7. Otherwise $\sigma^{d} \rightarrow \tau, d \rightarrow d+1$. If $d<n-1$, then $p=$ floor $\left(\frac{n+d}{2}\right)$ and go to Step 3. Otherwise go to Step 8;

645 Step 7: End, the $d$-ENOCF problem is solved;

Step 8: End, the $d$-ENOCF problem cannot be solved.

\section{Conclusion}

In this article, we have given a set of sufficient geometrical conditions that allow to transform a dynamical system into the $d$-Extended Nonlinear Canonical Form ( $d$-ENOCF) by dynamic extension. We started by proposing sufficient condition for a system to be transformed into the 1-ENOCF. Then, we proceeded step by step to generalise our methodology to the $d$-ENOCF. In particular, we showed that $d$ could not exceed $n-2$ for a state space of dimension $n$. Finally, we provided an algorithm for verifying whether the $d$-ENOCF problem is solvable.

\section{Acknowledgement}

The authors would like to thank all the reviewers for their useful and valuable comments in improving this article.

\section{Note}

1. This question was first raised by Professor Respondek at the IEEE Conference on Decision and Control (2005) in Seville during a nonlinear observer session.

\section{References}

Abraham, R., and Marsden, J.E. (1966), Foundation of Mechanics, New Jersey: Princeton.

Back, J., Yu, K.T., and Seo, J.H. (2005), 'Dynamic Observer Error Linearization', in Proceedings of IEEE CDC, 44, 6364-6369.

Back, J., Yu, K.T., and Seo, J.H. (2006), 'Dynamic Observer Error Linearization', Automatica, 42, 2195-2200.

Bestle, D., and Zeitz, M. (1983), 'Canonical form Observer Design for Nonlinear Time Variable Systems', International Journal of Control, 38, 419-431.

Boutat, D. (2007), 'Geometrical Conditions to Linearise Observer Error via $0,1, \ldots,(n-2)-\int$ ', in 7 th IFAC Symposium on Nonlinear Control Systems, Nolcos, 2007.

Busawon, K., and Kabore, P. (2001), 'Disturbance Attenuation using Proportional Integral Observers', International Journal of Control, 74, 618-627.

Fliess, M., and Kupka, I. (1983), 'A Finiteness Criterion for Nonlinear Input-Output Differential Systems', SIAM Journal of Control Optimisation, 21, 721-728.

Gauthier, J.P., Hammouri, H., and Othman, S. (1985), ‘A Simple Observer for Nonlinear Systems-application to Bioreactors', IEEE Transactions on Automatic Control, 37 , 875-880.

Glumineau, A., Moog, C.H., and Plestan, F. (1996), 'New Algebro-geometric Conditions for Linearization by Input-Output Injection', IEEE Transaction on Automatic Control, 41, 598-603.

Hammouri, H., and Gautier, J.P. (1988), 'Bilinearisation up to the Output Injection', System and Control Letters, 11, 139-149.

Hou, M., and Pugh, A.C. (1999), 'Observer with Linear Error Dynamics for Nonlinear Multi-output Systems', Systems and Control Letters, 37, 1-9.

Jouan, P. (2003), 'Immersion of Nonlinear Systems into Linear Systems Modulo Output Injection', SIAM Journal of Control and Optimization, 41, 1756-1778.

Keller, H. (1987), 'Nonlinear Observer Design by Transformation Into a Generalised Observer Canonical Form', SIAM Journal of Control and Optimization, 46, 1915-1930.

Krener, A.J., and Isidori, A. (1983), 'Linearization by Output Injection and Nonlinear Observer', Systems and Control Letters, 3, 47-52.

Krener, A, and Respondek, W. (1985), 'Nonlinear Observer with Linearisable Error Dynamics', SIAM Journal of Control and Optimization, 30, 197-216.

Noh, D., Jo, N., and Seo, H. (2004), 'Nonlinear Observer Design by Dynamic Observer Error Linearisation', IEEE Transactions on Automatica Control, , 49.

Phelps, A. (1991), 'On Constructing Nonlinear Observers', SIAM Journal of Control and Optimization, 29, 516-534. 
Respondek, W., Pogromsky, A., and Nijmeijer, H. (2004),

'Time Scaling for Observer Design with Linearisation Error Dynamics', Automatica, 40, 277-285.

Sontag, E.D. (1995), 'On the Input-to-state Stability Property', European Journal of Control, 1, 24-36.

Sontag, E.D., and Wang, Y. (1995), 'On Characterisations of the Input-to-state Stability Property', Systems Control Letters, 24, 351-359.

Xia, X.H., and Gao, W.B. (1989), 'Nonlinear Observer with Linearisable Error Dynamics', SIAM Journal of Control and Optimization, 27, 199-216.

\section{$730 \quad$ Appendix}

In this appendix we recall a useful technical formula (Abraham and Marsden 1966) from differential geometry which is used throughout this article. Additionally, the proof of Claim 2.1 is given.

735 Technical formula 1: Let $v$ be a differential 1-form. Its differential $d v$ is a 2-form which can be computed on two vector fields $X, Y$ as follows:

$$
\mathrm{d} v(X, Y)=L_{X} v(Y)-L_{Y} v(X)-v[X, Y] .
$$

If the 1-form $v$ is closed thus $\mathrm{d} v=0$ then the formula gives

$$
v[X, Y]=L_{X} v(Y)-L_{Y} v(X) .
$$

Moreover, if $L_{Y} v(X)=0$ then we have

$$
v[X, Y]=L_{X} v(Y) .
$$

From the construction of the Krener-Isidori frame $\tau$ (see Equations $(8,9)$ with $l=1)$, we can deduce the following facts:

$$
\begin{aligned}
\theta_{n-i}\left(\tau_{i+1}\right)=1 & \text { for } i=0, \ldots, n-1, \\
\theta_{n-j}\left(\tau_{k}\right)=0 & \text { for } 1 \leq k<j \leq n-1 .
\end{aligned}
$$

To obtain this relationship it is sufficient to use the formula (55) for $\nu=\theta_{n-1}, X=\tau_{1}, Y=\tau_{2}=\left[\tau_{1}, f\right]$ and the diagram given in Figure 2 which gives $\theta_{j+1}\left(\tau_{k}\right)=\theta_{j}\left(\tau_{k+1}\right)$ if $\theta_{j}\left(\tau_{k}\right)=0$. Now, since $\theta_{n-1}\left(\tau_{1}\right)=\theta_{n-2}\left(\tau_{1}\right)=0, \theta_{n}\left(\tau_{1}\right)=1$ then $\theta_{n-1}\left(\tau_{2}\right)=1$ and $\theta_{n-2}\left(\tau_{2}\right)=0$. The rest is obtained by induction and by using the same argument.
Proof of Claim 2.1: By definition, we have

$$
\sigma_{2}=\left[\sigma_{1}, f\right]=\left[l(y) \tau_{1}, f\right]=l(y)\left[\tau_{1}, f\right]-L_{f} l \tau_{1} .
$$

On the other hand, $\left[\tau_{1}, f\right]=\tau_{2}$ hence $\sigma_{2}=l(y) \tau_{2}-L_{f} f \tau_{1}$. As a result (13) is true.

Now we prove (14) by induction. For this, suppose that the formula (14) is true for a certain index $k$ and show that it is also true for $k+1$. For this, suppose that $\sigma_{k}=$ $\sum_{i=0}^{k-1}(-1)^{i} \Gamma_{k}^{i}\left(L_{f}^{i} l\right) \tau_{k-i}$, then

$$
\sigma_{k+1}=\left[\sigma_{k}, f\right]=\left[\sum_{i=0}^{k-1}(-1)^{i} \Gamma_{k}^{i}\left(L_{f}^{i} l\right) \tau_{k-i}, f\right] .
$$

As $\left[\left(L_{f}^{i} l\right) \tau_{k-i}, f\right]=\left(L_{f}^{i} l\right) \tau_{k-i+1}-\left(L_{f}^{i+1} l\right) \tau_{k-i}$, therefore we have

$$
\sigma_{k+1}=\sum_{i=0}^{k-1}(-1)^{i} \Gamma_{k}^{i}\left(\left(L_{f}^{i} l\right) \tau_{k-i+1}-\left(L_{f}^{i+1} l\right) \tau_{k-i}\right) .
$$

Now, the second term of this sum can be written as

$$
\left.\left.\sum_{i=0}^{k-1}(-1)^{i+1} \Gamma_{k}^{i}\left(L_{f}^{i+1} l\right) \tau_{k-i}\right)=\sum_{i=1}^{k}(-1)^{i} \Gamma_{k}^{i-1}\left(L_{f}^{i} l\right) \tau_{k-i+1}\right) .
$$

Hence

$$
\begin{aligned}
\sigma_{k+1}= & \Gamma_{k}^{0} l \tau_{k+1}+\sum_{i=1}^{k}(-1)^{i}\left(\Gamma_{k}^{i}+\Gamma_{k}^{i-1}\right)\left(L_{f}^{i} l\right) \tau_{k+1-i} \\
& -(-1)^{k} \Gamma_{k}^{k}\left(L_{f}^{k} l\right) \tau_{1} \\
= & l \tau_{k+1}+\sum_{i=1}^{k}(-1)^{i}\left(\Gamma_{k}^{i}+\Gamma_{k}^{i-1}\right)\left(L_{f}^{i} l\right) \tau_{k+1-i} .
\end{aligned}
$$

Finally, since $\Gamma_{k+1}^{i}=\Gamma_{k}^{i}+\Gamma_{k}^{i-1}$, we obtain the desired formula

$$
\begin{aligned}
\sigma_{k+1} & =l \tau_{k+1}+\sum_{i=1}^{k}(-1)^{i} \Gamma_{k+1}^{i}\left(L_{f}^{i} l\right) \tau_{k+1-i} \\
& =\sum_{i=0}^{k}(-1)^{i} \Gamma_{k+1}^{i}\left(L_{f}^{i} l\right) \tau_{k+1-i},
\end{aligned}
$$

thus we prove the Claim 2.1 by induction. 